\title{
Plasmasphere Modeling with Ring Current Heating
}

\author{
S. M. Guiter ${ }^{1}$, M.-C. Fok ${ }^{1}$, and T. E. Moore \\ Space Sciences Laboratory, NASA Marshall Space Flight Center, Huntsville, Alabama
}

\begin{abstract}
Coulomb collisions between ring current ions and the thermal plasma in the plasmasphere will heat the plasmaspheric electrons and ions. During a storm such heating would lead to significant changes in the temperature and density of the thermal plasma. This was modeled using a time dependent, one-stream hydrodynamic model for plasmaspheric flows, in which the model flux tube is connected to the ionosphere. The model simultaneously solves the coupled continuity, momentum, and energy equations of a two-ion $\left(\mathrm{H}^{+}\right.$and $\left.\mathrm{O}^{+}\right)$quasineutral, currentless plasma. Heating rates due to collisions with ring current ions were calculated along the field line using a kinetic ring current model. First, diurnally reproducible results were found assuming only photoelectron heating of the thermal electrons. Then results were found with heating of the $\mathrm{H}^{+}$ions by the ring current during the recovery phase of a magnetic storm.
\end{abstract}

\section{INTRODUCTION}

Significant heating of the thermal plasma in the plasmasphere can result from interactions with ring current ions. This was studied by Chandler et al. [1988] using the FLIP model; for this work the thermal plasma was assumed to be heated by Coulomb collisions with the ring current ions, with equatorial heating rates calculated using the methods of Kozyra et al. [1987] and with a Gaussian profile in latitude. More recently, Gorbachev et al. [1992] investigated the heating of the thermal plasma by MHD waves which would be generated by the ring current; they used a hydrodynamic model which included the heat flow equations.

For this work the heating of $\mathrm{H}^{+}$ions due to Coulomb collisions with ring current ions during the recovery phase of a magnetic storm was studied. Heating rates per particle were derived from a three-dimensional kinetic ring current model [Fok et al., 1995a]. A time-dependent hydrodynamic plasmasphere model was used; the magnetic field was assumed to be a corotating dipole. It is fully inter-

INAS/NRC Research Associate.

Cross-Scale Coupling in Space Plasmas

Geophysical Monograph 93

This paper is not subject to U.S. copyright. Published in 1995

by the American Geophysical Union hemispheric and no diffusive equilibrium assumptions are made.

\section{MODEL DESCRIPTION}

For this work the model is essentially the same as de scribed in Guiter et al. (Modeling of $\mathrm{O}^{+}$ions in the plasmasphere, submitted to J. Geophys. Res., 1995), with some modifications. It includes the time-dependent continuity, momentum, and energy equations for $\mathrm{O}^{+}$and $\mathrm{H}^{+}$ions, and the energy equation for electrons; the plasma is assumed to be quasineutral and currentless [cf. Guiter and Gombosi, 1990]. Ionization, charge exchange, recombination, collisions, and heat conduction are included; external heat sources are allowed. Photoelectron heating of thermal elec trons is described using analytic formulas which include the effect of trapped photoelectrons. Neutral parameters were found using the MSIS-86 [Hedin, 1987] (densities and temperatures) and HWM-90 [Hedin et al., 1991] (winds) models, for February 9, 1986. Also, the energy loss of $\mathrm{H}^{+}$ions due to charge exchange with $\mathrm{H}$ atoms was included. This was calculated using the cross section for this process as a function of energy, as given in Barnett [1990].

The decay of the ring current during the February, 1986 storm was simulated using a kinetic ring current model [Fok et al., 1995a]. In this model, the distribution function is found as a function of pitch angle and energy; drifts, and losses due to charge exchange and Coulomb collisions are 
included. The initial distributions of the ring current species are found by extrapolating from AMPTE/CCE observations on the dawn and dusk sides of the inner magne tosphere; boundary values on the nightside are taken from CCE ion flux data at $L=6.75$ during the storm. The ring current fluxes along the field line are inferred from the equatorial distribution function [cf. Fok et al., 1995a]. The simulation began at 0300 UT on February 9,1986 . At various times in the simulation average heating rates per particle were calculated, as a function of local time, $L$ shell, and magnetic latitude. It was assumed that only $\mathrm{H}^{+}$ions were heated by the ring current interactions, as the heating rate per particle for $\mathrm{O}^{+}$is much smaller than that for $\mathrm{H}^{+}$ (personal communication, M.-C. Fok, 1995). For $L-2$ and $L=3$, these heating rates were set to zero for magnetic latitudes greater than $\mathbf{4 0}$ degrees, which is the boundary of the ring current model for these $L$ shells.

For the plasmasphere modeling, a longitude was chosen so that $0 \mathrm{LT}$ would be approximately equal to $0300 \mathrm{UT}$; the magnetic longitude was set to 25 degrees. For the $L=2$ flux tube, the model was run for several days assuming only photoelectron heating, until the results were approximately diurnally reproducible. Then the model was allowed to run for one day with the ring current heating of the $\mathrm{H}^{+}$ions included. For the $L=3$ flux tube, results with ring current heating were found starting from a solution which corresponds to a partially filled flux tube.

\section{RESULTS}

Figure 1 shows results found when the model was run on an $L=2$ flux tube. The solid curve shows results found assuming photoelectron heating only, the dotted line gives results when the ring current heating started at $0 \mathrm{LT}$, and the dashed line gives results when the ring current heating started at $1200 \mathrm{LT}$. For the case when the heating starts at $0 \mathrm{LT}$ the equatorial $\mathrm{H}^{+}$temperature rises to a value of about $35,000 \mathrm{~K}$ after 4 hours and then decreases more gradually; the equatorial $\mathrm{H}^{+}$density drops as the temperature increases. For this case the maximum ion heating rate per particle is about $2 \times 10^{-3} \mathrm{eV} / \mathrm{s}$. The equatorial temperature increase causes downward flows due to the increased pressure gradients, with $\mathrm{H}^{+}$velocities reaching $10 \mathrm{~km} / \mathrm{s}$ near $500 \mathrm{~km}$ altitude (velocity profiles not shown). In the case when the ring current heating starts at $1200 \mathrm{LT}$ the temperature does not change much until 12 hours into the simulation, when the temperature starts to rise. At this time the flux tube is on the nightside, which is where the ring current heating rates are highest. The equatorial $\mathrm{H}^{+}$temperature only rises to about $22,000 \mathrm{~K}$ in this case.

Figure 2 shows results found on an $L-3$ flux tube; the solid line gives results found assuming photoelectron heat ing only, while the dotted line gives results when the ring current heating was included and assumed to start at $0 \mathrm{LT}$. In the first four hours $\mathrm{T}_{\mathrm{eq}}\left(\mathrm{H}^{+}\right)$rises to about $12,000 \mathrm{~K}$, and rises again in the afternoon, reaching a maximum of about
$20,000 \mathrm{~K}$. In addition, the plasmasphere density is lower when the ring current heating is included.

\section{DISCUSSION AND SUMMARY}

We have given results found using a time-dependent plasmasphere model when ring current heating of the $\mathrm{H}^{+}$ ions is included. It was found that this would lead to significant enhancements of the equatorial $\mathrm{H}^{+}$temperature and that the equatorial $\mathrm{H}^{+}$density decreases when the temperature rises. A similar study [Fok et al., 1995b] was done using the FLIP model; they found a similar time profile for the ion temperature, although the maximum temperature in their results is only about $14,000 \mathrm{~K}$ on an $L=2$ field line. This difference is probably a result of the assumption in the
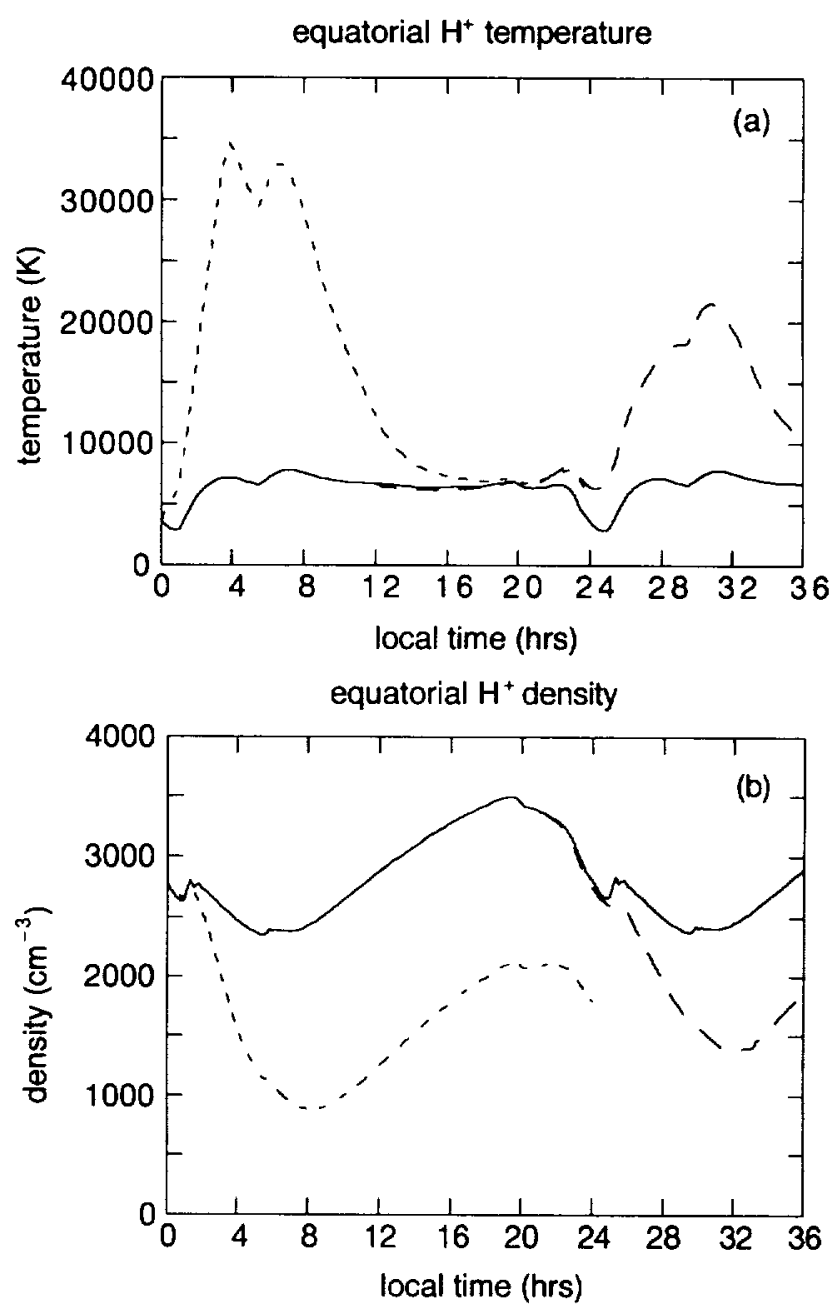

Fig. 1. Local time profiles of the equatorial $\mathbf{H}^{+}$temperature (a) and density (b) for $L=2$. The solid line shows results when only photoelectron heating of electrons is included; the dotted line gives results when the ring current heating of $\mathrm{H}^{+}$ions started at 0 LT; the dashed line gives results when the ring current heating of $\mathrm{H}^{+}$ions started at $1200 \mathrm{LT}$. 

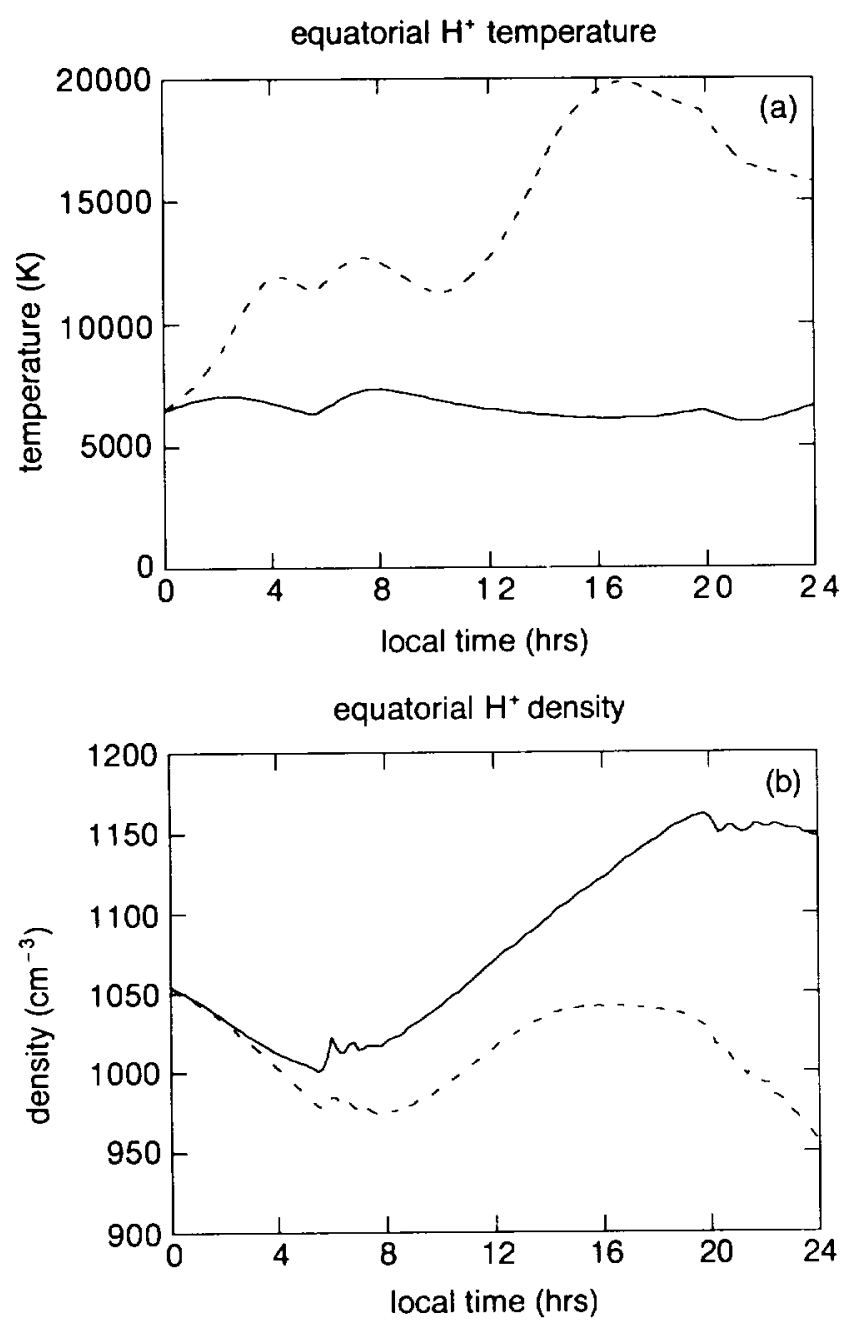

Fig. 2. Local time profiles of the equatorial $\mathrm{H}^{+}$temperature (a) and density (b) for $L=3$. The solid line shows results when only photoelectron heating of electrons is included; the dotted line gives results when the ring current heating of $\mathrm{H}^{+}$ions started at 0 LT.

FLIP model that there is only one ion temperature, whereas in our plasmasphere model an energy equation is solved for each species. Assuming that the different ion species have the same temperature implies that collisions are strong enough to keep them in thermal equilibrium, and that the per particle heating rates are the same for $\mathrm{H}^{+}$and $\mathrm{O}^{+}$. However, the per particle heating rates due to ring current interactions are roughly twenty times larger for $\mathrm{H}^{+}$ than for $\mathrm{O}^{+}$(personal communication, M.-C. Fok, 1995), and the momentum transfer collision frequency of $\mathrm{H}^{+}$with $\mathrm{O}^{+}$is inversely proportional to the $\mathrm{H}^{+}$temperature raised to the power 1.5 , which means that the two ion species should rapidly be thermally decoupled. Furthermore, to force the ion temperatures to be the same in our model would re quire an enhancement of the thermal coupling between $\mathrm{H}^{+}$ and $\mathrm{O}^{+}$, which would be equivalent to extra energy loss for the $\mathrm{H}^{+}$ions.

The temperature rise is probably unrealistically high in our $L=2$ results. This could be a result of the ion heating rates being too high; changing the method for obtaining the initial ion fluxes at energies lower than the lowest energy measured by AMPTE can reduce the heating rates by an order of magnitude at lower $L$ shells [cf. Fok et al., 1995b]. With the ion heating rates reduced by a factor of ten, the maximum equatorial $\mathrm{H}^{+}$temperature is about $10,000 \mathrm{~K}$ for $L=2$. These results show that after the main phase of a magnetic storm ring current heating would have a significant effect on the thermal plasma in the plasmasphere.

Acknowledgments. This work was performed while two of the authors (S. M. Guiter and M.-C. Fok) held National Research Council-Marshall Space Flight Center Research Associateships. Acknowledgment is also made to Dr. Paul Craven for helpful discussions.

\section{REFERENCES}

Barnett, C. F., Atomic Data for Fusion, vol. 1: Collisions of $H$, $\mathrm{H} 2$, He, and Li Atoms and lons with Atoms and Molecules, Technical Report ORNL-6086/V1, Oak Ridge National Laboratory, Oak Ridge, TN, 1990.

Chandler, M. O., J. U. Kozyra, J. L. Horwitz, R. H. Comfort, and L. H. Brace, Modeling of the thermal plasma in the outer plasmasphere -- a magnetospheric heat source, in Modeling Magnetospheric Plasma, Geophys. Monogr. Sr., vol. 44, ed. by T. E. Moore and J. H. Waite, Jr., pp. 101-105, AGU, Washington, D. C., 1988.

Fok, M.-C., T. E. Moore, J. U. Kozyra, G. C. Ho, and D. C. Hamilton, Three-dimensional ring current decay model, $J$. Geophys. Res., 100, 9619, 1995a.

Fok, M.-C., P. D. Craven, T. E. Moore, and P. G. Richards, Ring current-plasmasphere coupling through Coulomb collisions, this monograph, $1995 \mathrm{~b}$.

Gorbachev, O. A., G. V. Khazanov, K. V. Gamayunov, and E. N. Krivorutsky, A theoretical model for the ring current interaction with the earth's plasmasphere, Planet. Space Sci., $40,859,1992$.

Guiter, S. M., and T. I. Gombosi, The role of high speed plasma flows in plasmaspheric refilling, J. Geophys. Res., 95, 10,427, 1900.

Hedin, A. E., MSIS-86 thermospheric model, J. Geophys. Res., $92,4649,1987$.

Hedin, A. E., M. A. Biondi, R. G. Bumside, G. Hernandez, R. M. Johnson, T. L. Killeen, C. Mazaudier, J. W. Meriwether, J. E. Salah, R. J. Sica, R. W. Smith, N. W. Spencer, V. B. Wickwar, and T. S. Virdi, Revised global model of thermosphere winds using satellite and ground-based observations, J. Geophys. Res., 96, 7657, 1991.

Kozyra, J. U., E. G. Shelley, R. H. Comfort, L. H. Brace, T. E. Cravens, and A. F. Nagy, The role of ring current $\mathrm{O}^{+}$in the formation of stable auroral red arcs, J. Geophys. Res., 92, 7487, 1987.

S. M. Guiter, M.-C. Fok, and T. E. Moore, Space Sciences Laboratory, NASA Marshall Space Flight Center, Huntsville, AL 35812 . 
